\title{
Médiévales
}

Langues, Textes, Histoire

70 | printemps 2016

Lieux d'hygiène et lieux d'aisance en terre d'Islam (VII $\mathrm{e}_{-}$ $\mathrm{XV}^{\mathrm{e}}$ siècle)

\section{Latrines et espaces d'ablution dans les mosquées du Proche-Orient médiéval : l'enseignement des fouilles de Tyr}

Latrines and Ablution Places in the Mosques of medieval Middle-East: the Contribution of Tyre Excavations

\section{Marie-Odile Rousset}

\section{OpenEdition}

\section{Journals}

Édition électronique

URL : http://journals.openedition.org/medievales/7811

DOI : 10.4000/medievales.7811

ISSN : 1777-5892

\section{Éditeur}

Presses universitaires de Vincennes

\section{Édition imprimée}

Date de publication : 23 juin 2016

Pagination : 105-127

ISSN : 0751-2708

\section{Référence électronique}

Marie-Odile Rousset, «Latrines et espaces d'ablution dans les mosquées du Proche-Orient médiéval l'enseignement des fouilles de Tyr », Médiévales [En ligne], 70 I printemps 2016, mis en ligne le 15 juin 2018, consulté le 19 avril 2019. URL : http://journals.openedition.org/medievales/7811 ; DOI : $10.4000 /$ medievales.7811 


\section{Latrines et espaces d'ablution dans les mosquées du Proche-Orient médiéval: l'enseignement des fouilles de Tyr}

Selon les légistes musulmans, l'une des conditions prérequises de la prière est d'être en état de pureté rituelle. Il s'agit de la pureté prescrite (par opposition à la pureté réelle), obtenue par l'ablution simple ou totale qui annulent respectivement l'impureté mineure et majeure ${ }^{1}$.

L'obligation de se purifier avant la prière est clairement évoquée dans le Coran (V, 6 et IV, 43). Le déroulement du rituel des ablutions simples (wudu $\left.\bar{u}^{\prime}\right)$ selon les sunnites shafi'ites comporte six points : l'expression de l'intention, le lavage du visage, celui des deux mains jusqu'aux coudes, la friction du crâne, le lavage des deux pieds et le respect de l'ordre d'accomplissement de ces opérations ${ }^{2}$. Cependant, le nombre de lavages et l'importance de l'ordre dans lequel les choses se font diffèrent d'une école juridique à l'autre ${ }^{3}$. Le ghusl est le lavage de la totalité du corps, y compris les cheveux, avec de l'eau pure, après en avoir déclaré l'intention ${ }^{4}$. La tradition veut que les mains et parties génitales soient lavées, que les matières impures soient ôtées du corps et que de l'eau soit versée sur le corps depuis la tête jusqu'aux pieds trois fois. Sur cette question, les écoles

1. La pureté réelle exige quant à elle l'élimination de toute souillure du corps, du vêtement ou du lieu. Voir l'article de Yassir Benhima dans ce même volume, ainsi que les articles de l'Encyclopédie de l'Islam, Leyde/Paris, 1960-2009 (ci-dessous abrégée EI2) : șalāt (prière), wudū' (purification mineure), țahāra (pureté rituelle), nadjiis (impur, opposé de tahāra), hadath (impureté rituelle mineure), djanāba (impureté majeure), ghusl (purification majeure).

2. «wud̄" $\bar{u}^{\prime} », E I 2$.

3. A. KEVIN REINHART, «Impurity/No Danger », History of Religions, 30 (1990), p. 1-24 (p. 12-13).

4. «ghusl»,EI2. 
juridiques ont aussi chacune leurs règles auxquelles s'ajoutent des pratiques variées ${ }^{5}$.

Il est communément admis que la purification majeure ${ }^{6}$ se faisait dans les bains privés ou publics tandis que le lieu de la purification mineure était généralement associé à des latrines qui devaient être proches de la mosquée ${ }^{7}$. Ces espaces n'ont, à ce jour, que rarement retenu l'attention des archéologues et des historiens de l'architecture ${ }^{8}$, même si on peut noter une évolution de cette question chez certains chercheurs ${ }^{9}$. Annexes à l'édifice religieux proprement dit, entachées d'un certain tabou, parfois mal interprétées, ces constructions sont aussi d'un abord difficile car elles ont été et sont - dans le cas d'édifices encore en activité - l'objet de nombreuses réfections ou reconstructions. Pour contribuer à l'étude de ces installations, cet article présente un cas récemment étudié, à Tyr, et envisage ses particularités au regard des autres exemples du Moyen-Orient médiéval.

\section{Salle d'ablution et latrines de la grande mosquée de Tyr}

À Tyr, les fouilles de la Direction générale des antiquités libanaises dans le secteur de la cathédrale, dirigées par Maurice Chéhab vers le début des années 1970, ont mis au jour un édifice trapézoïdal, aligné avec un grand mur de fondation nord-sud (fig. 1) ${ }^{10}$. Ces constructions, antérieures à l'édification de la cathédrale par les Croisés, n'ont pas retenu l'attention des fouilleurs et n'ont pas été publiées. Les travaux archéologiques réalisés au cours de la campagne $2012^{11}$, suite à la redécouverte de ce «bâtiment

5. A. KEVIN REINHART, «Impurity/No Danger », p. 16 : comme dans le cas du wudü', les parties du corps concernées par l'impureté qu'il faut effacer et celles qui sont abluées ne sont pas forcément les mêmes.

6. « hammām», EI2.

7. Le terme miḍa'a (Encyclopédie de l'Islam) est plus couramment utilisé en al-Andalus et au Maghreb. Il désigne parfois également une fontaine pour les ablutions au centre de la cour de la mosquée.

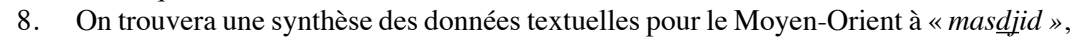
chapitre «alimentation en eau », EI2. À compléter par M. MEINECKE, Die mamlukische Architektur in Ägypten und Syrien (648/1250 bis 923/1517), Glückstadt, 1992, qui donne la liste des fondations/constructions d'après les mentions textuelles et les inscriptions. En effet, ce genre d'édifice donne souvent lieu à des fondations pieuses, répertoriées dans les actes de waqf.

9. Par exemple J.-C. GolvIN, Essai sur l'architecture religieuse musulmane, Paris, vol. I, 1970, chapitre VIII, « bâtiments annexes », p. 250-252 (deux pages sur les « salles d'ablution » et une seule phrase sur les latrines) ; ID., La Madrasa médiévale, Aix-enProvence, 1995, où il présente systématiquement, lorsqu'elles sont conservées, les salles d'ablution.

10. Cette photographie, prise à la fin des fouilles dans ce secteur, est reproduite ici avec l'aimable autorisation de la DGA libanaise que je remercie.

11. Dans le cadre de la mission archéologique libano-française de Tyr, sous la direction de Ali Badawi (DGA) et Pierre-Louis Gatier (CNRS), une étude plus spécifique porte sur 


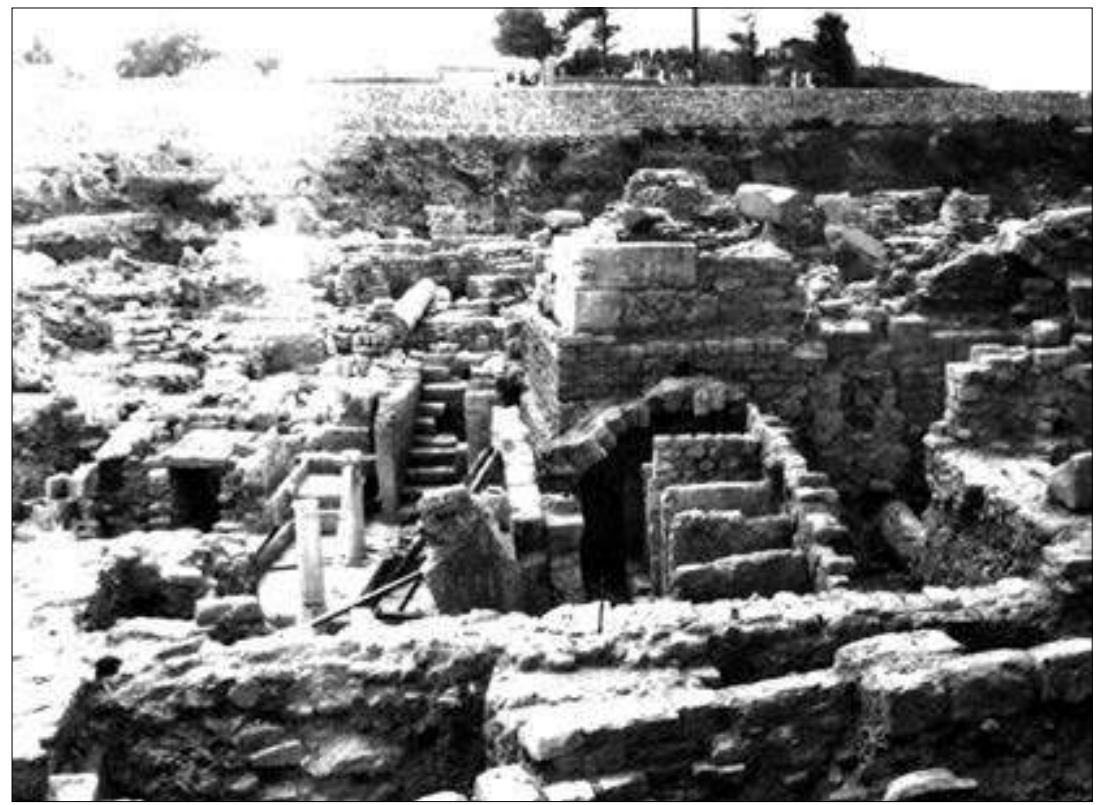

Figure 1. Vue générale de la fouille à l'époque de Maurice Chéhab, au début des années 1970

(C) Archives DGA Liban)

à bassin ${ }^{12}$, ont montré qu'il s'agissait d'un espace réservé aux ablutions rituelles et aux lieux d'aisance, associé à la mosquée congrégationnelle de $\operatorname{Tyr}^{13}$.

Une grande citerne qui prolonge le bras nord du transept de l'église empiète sur l'angle sud-ouest de l'édifice et fournit une relation stratigraphique indubitable entre les constructions musulmane et croisée. Même si on ne connaît pas exactement la date de cette dernière, elle ne peut pas être antérieure à la prise de la ville par les Latins en 1124, terminus

le secteur dit « de la cathédrale », sous la responsabilité d'Anne Baud (Université Lumière Lyon 2). L'équipe qui a étudié l'espace d'ablution en 2012-2013 était composée de Patrick Ferreira (Inrap Méditerranée), Claudine Piaton (INHA), Marie Rochette (Inrap Méditerranée), Marie-Odile Rousset (CNRS, Gremmo) et Apolline Vernet (Université Paris I).

12. P.-L. Gatier, A. Baud, D. Cahu, G. Charpentier, A. Devillechaise, C. Duvette, M. El-Masri Hachem, P. Ferreira, A. Flammin, N. Haidar-Vela, X. Husson, H. KahWAGi-JANHo, C. Piaton, D. PIERI et A. SChMitT, « Mission archéologique de Tyr. Rapport Préliminaire 2008-2009 », Baal, 14 (2010), p. 135-240 (p. 166-187, plan fig. 37).

13. Cette mosquée, sous la cathédrale de Tyr, n'avait jamais été évoquée avant notre étude car elle a été complètement démolie lors de la construction de la cathédrale. La présence de ce bâtiment d'ablution est l'un des arguments de son identification. Le secteur ayant été fouillé à plusieurs reprises, il n'en reste que quelques vestiges. Ceux-ci seront publiés dans P.L. GATIER éd., Mission archéologique de Tyr (2008-2013), Baal, Supplément n 14, Beyrouth. 
antequem pour la datation de la mosquée. Les textes mentionnent peu de choses sur la ville du début de l'époque islamique, excepté ses fortifications, qui semblent être cantonnées au port et à une surface équivalente au sud (d'après al-Muqaddasī, seconde moitié du X $\mathrm{X}^{\mathrm{e}}$ siècle), c'est-à-dire en dehors du secteur de la cathédrale qui se trouve au centre de la ville médiévale. En revanche, les sources montrent que la ville de Tyr s'est fortement développée au cours de la période fatimide (fin du X ${ }^{\mathrm{e}}-\mathrm{XII}^{\mathrm{e}}$ siècle) ${ }^{14}$. La mosquée et son bâtiment d'ablution pourraient donc avoir été construits entre la fin du $\mathrm{X}^{\mathrm{e}}$ et le début du $\mathrm{XI}^{\mathrm{e}}$ siècle, ce qui en ferait le plus ancien complexe de ce type mis au jour au Proche-Orient.

Le nettoyage et l'analyse des appareils ont permis de dresser le plan de ce bâtiment et mis en évidence deux grandes phases de construction et d'utilisation. Seules de rares couches en place ont pu être fouillées, la zone ayant été entièrement décaissée lors des interventions précédentes. L'édifice, d'environ 12 m nord-sud par 9 m est-ouest, a été en partie enterré dans les niveaux préexistants dont il a coupé les vestiges (par exemple, les boutiques de l'Antiquité tardive). La construction a été insérée dans l'angle formé par deux murs antérieurs qui ont servi de soutènement. Les parements intérieurs des murs périphériques ont été plaqués contre les couches coupées par le décaissement, sauf à l'est, où le mur forme le côté d'un grand égout nord-sud. Celui-ci mesure 1,20 m de hauteur pour 0,60 m de largeur et ses dalles de couverture sont prises dans ce mur à la phase II. Cependant, son état primitif pourrait être antérieur au bâtiment et aurait conditionné l'orientation de ce mur est.

\section{Le bâtiment d'ablution primitif (phase l)}

Le plan du bâtiment s'organise autour d'un espace central dallé (A), auquel on accédait au moyen d'un escalier ménagé du côté sud (fig. 2 et 3). Le dallage présente deux orientations et deux niveaux distincts de part et d'autre d'une ligne nord-sud matérialisée, dans sa partie nord, par un mur, plus tard arasé.

Une dizaine de petits espaces, de 0,60 m de profondeur par 0,50 m de largeur, aménagés à l'intérieur du mur oriental, s'ouvrent sur la cour. Chacun est voûté et enduit de mortier. Leur sol était sans doute plus bas que le seuil, en petites colonnes de marbre remployées, qui court devant l'ensemble (fig. 4 et 5).

Comme le montre un exemple conservé au nord, une niche de $0,30 \mathrm{x}$ 0,30 × $0,30 \mathrm{~m}$ était aménagée au-dessus de chacun d'eux pour protéger

14. D. Bramoullé, « Tyr dans les sources de la période fatimide (969-1171) », dans P.-L. Gatier, J. AliQuot et L. Nordiguian éd., Sources de l'histoire de Tyr : textes de l'Antiquité et du Moyen Âge, Beyrouth, 2011, p. 157-177. 




Figure 2. Vue générale de la partie est après la fouille de 2012, depuis le nord (Cliché Marie-Odile Rousset (C) Mission archéologique de Tyr)

les chaussures et autres affaires des croyants pendant leurs ablutions. Cet ensemble est interrompu, au nord, par une porte étroite $(0,40 \mathrm{~m})$ surmontée d'un arc, qui donnait accès à l'égout.

Contre le mur nord, une série d'au moins six compartiments rectangulaires de 1,80 m x 0,80 m étaient juxtaposés (I, N, J et K, fig. 6).

Celui qui se trouvait dans la pièce $\mathrm{H}$ a été détruit par le réaménagement postérieur, tout comme le plus oriental, pris dans la maçonnerie d'un massif ottoman. Il s'agit d'un système mixte, associant latrines et espaces d'ablutions. Chaque cabinet est accessible par une porte au sud et était couvert d'une voûte en berceau. Une rigole court à la base du mur nord, sur l'ensemble des cabines. Composée de blocs de grès $(0,28 / 0,30 \times 0,17 \times 0,12 \mathrm{~m})$ creusés d'une rainure en « $\mathrm{U}$ » (env. 0,07 x $0,03 \mathrm{~m}$ ), et recouverts d'enduit blanc, elle traverse chaque mur de refend, une pierre servant de linteau. Dans chaque pièce, au nord-ouest, un petit bassin creusé dans un bloc de grès (cuvette : 0,06 $\mathrm{m}$ de profondeur par $0,13 \times 0,10 \mathrm{~m}$ ) récupérait l'eau du canal via une petite fente. L'eau pouvait ainsi être puisée directement dans le bassin, sans souiller celle qui s'écoulait dans le canal (fig. 7).

En avant, et parallèle à ce dispositif, est disposée la latrine : elle est bordée, côté nord, par une pierre rectangulaire et, côté sud, par une dalle ou un massif maçonné qui occupe la moitié orientale de la pièce. La partie 

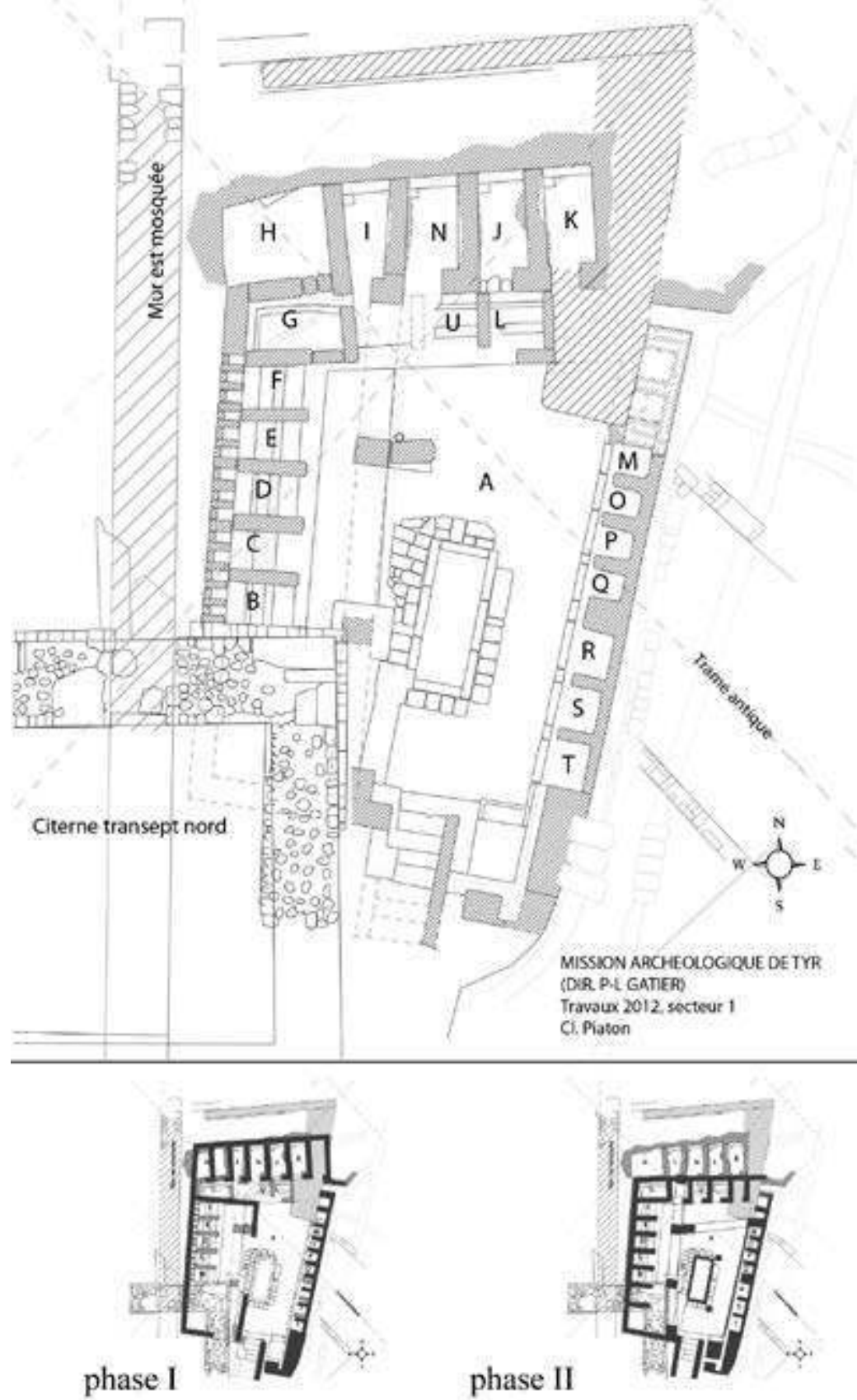

Figure 3. Plan général du secteur d'ablution de la mosquée de Tyr (Claudine Piaton () Mission archéologique de Tyr) 


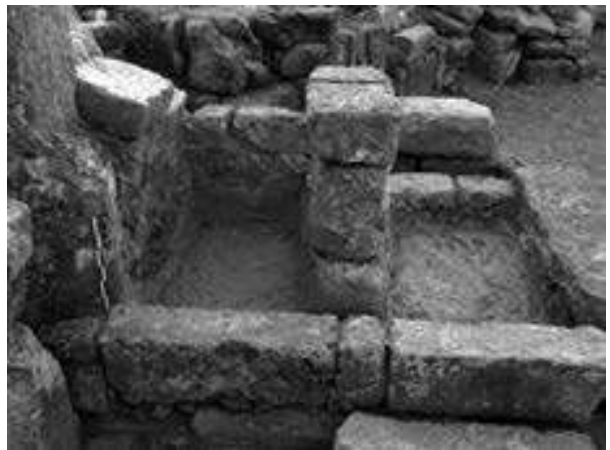

Figure 4.

Compartiments

$\mathrm{M}$ et $\mathrm{O}$ de la phase $\mathrm{I}$,

vus de l'ouest

(Cliché Marie-Odile Rousset

(C) Mission archéologique de Tyr)
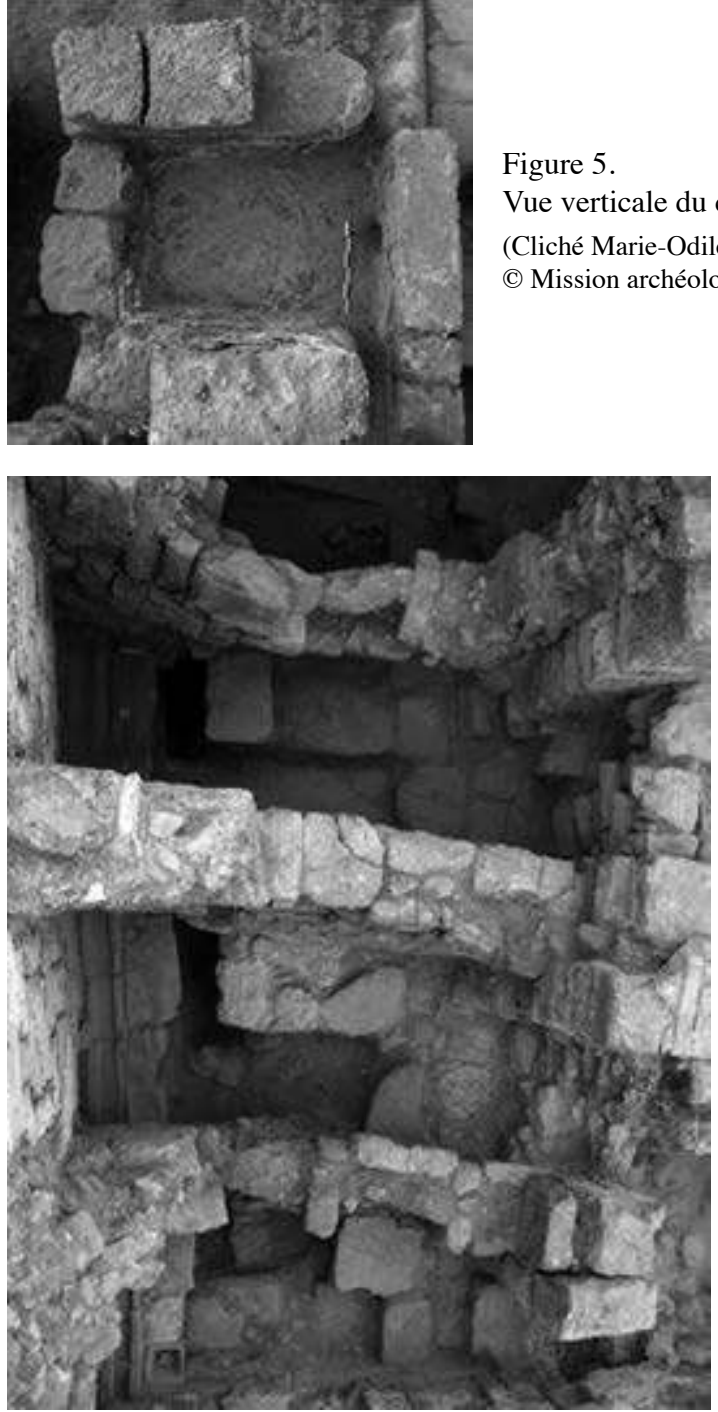

Figure 5.

Vue verticale du compartiment $\mathrm{O}$ de la phase I

(Cliché Marie-Odile Rousset

(C) Mission archéologique de Tyr)
Figure 6.

Cabinets I à K

contre le mur nord

(Cliché Marie-Odile Rousset

(C) Mission archéologique de Tyr) 


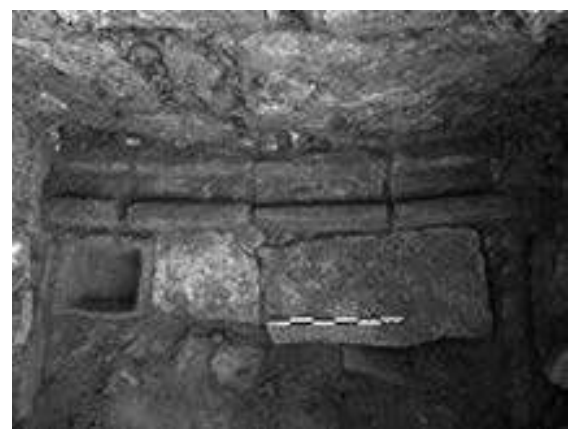

Figure 7. L'adduction d'eau pure de la phase I, au fond du compartiment I

(Cliché Marie-Odile Rousset @ Mission archéologique de Tyr)

occidentale est occupée par un bac enduit, d'une dizaine de centimètres de profondeur, dont l'eau s'évacuait par le trou des latrines, dans une canalisation en contrebas. Les murs de ces pièces étaient recouverts de trois couches d'enduit blanc : la première porte des traces de traits peints en noir (il s'agit peut-être d'inscriptions ; on distingue un «ayn»), la deuxième porte des décors gravés de type graffiti; la troisième est partiellement peinte en rouge, avec, comme sur le mur oriental de la pièce $\mathrm{N}$, des motifs peints. De nombreux fragments d'enduits rouges ont été retrouvés dans la couche de remblai qui recouvrait ce niveau.

Le mur ouest du bâtiment est lui aussi recouvert de trois couches d'enduits blancs successifs, le deuxième peint en rouge. Ils attestent d'un premier état dans ce secteur, qui a été masqué par les réaménagements tardifs. Le mur est construit avec des blocs de grès de taille moyenne $(0,21 \mathrm{~m}$ x $0,29 \mathrm{~m}$ $\mathrm{x} 0,12 \mathrm{~m}$ ), relativement réguliers, probablement des remplois provenant des boutiques antiques. À l'intérieur du mur court une canalisation en « $\mathrm{U}$ » large de 0,16 à $0,19 \mathrm{~m}$, entièrement enduite, en pente vers le nord. Sur son parement est, des ouvertures quadrangulaires $(0,23 \times 0,25 \mathrm{~m})$, également couvertes d'enduit lisse, sont régulièrement aménagées, donnant accès à l'eau qui devait être puisée au moyen de petits godets (fig. 8).

Certaines d'entre elles ont été condamnées lors de l'édification des murets de séparation de la phase II. Enfin, dans cette partie, le dallage de la pièce A venait buter contre le côté est d'un drain, ce qui permet de restituer là des latrines dès la phase I. Étant donné la hauteur du canal interne de ce mur ouest par rapport au sol de la pièce $\mathrm{A}(0,90 \mathrm{~m})$, il devait déjà y avoir, à l'origine, une sorte de banquette aménagée contre ce mur, au-dessus du canal d'évacuation. À ce premier état pourrait appartenir également le mur nord-sud, qui subsiste uniquement à son niveau d'arasement dans le dallage de la cour qui s'appuie contre lui (il a été détruit par la construction d'une canalisation à la phase II). Parallèle à l'orientation générale de l'aile ouest du bâtiment, il définissait un couloir, peut-être couvert, qui desservait les latrines occidentales et les isolait du reste de la cour (fig. 9). 


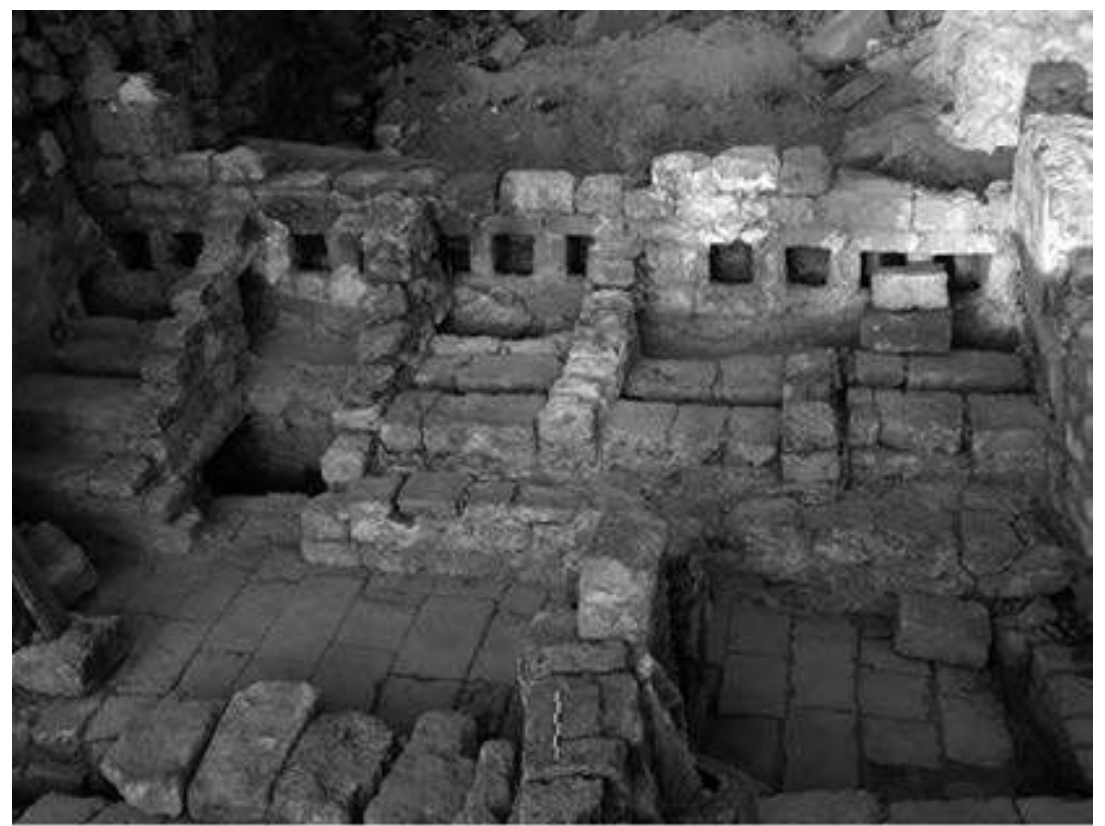

Figure 8. Latrines $\mathrm{B}$ à $\mathrm{F}$ de la phase II

Dans le mur, le canal d'eau pure de la phase I.

(Cliché Marie-Odile Rousset (c) Mission archéologique de Tyr)

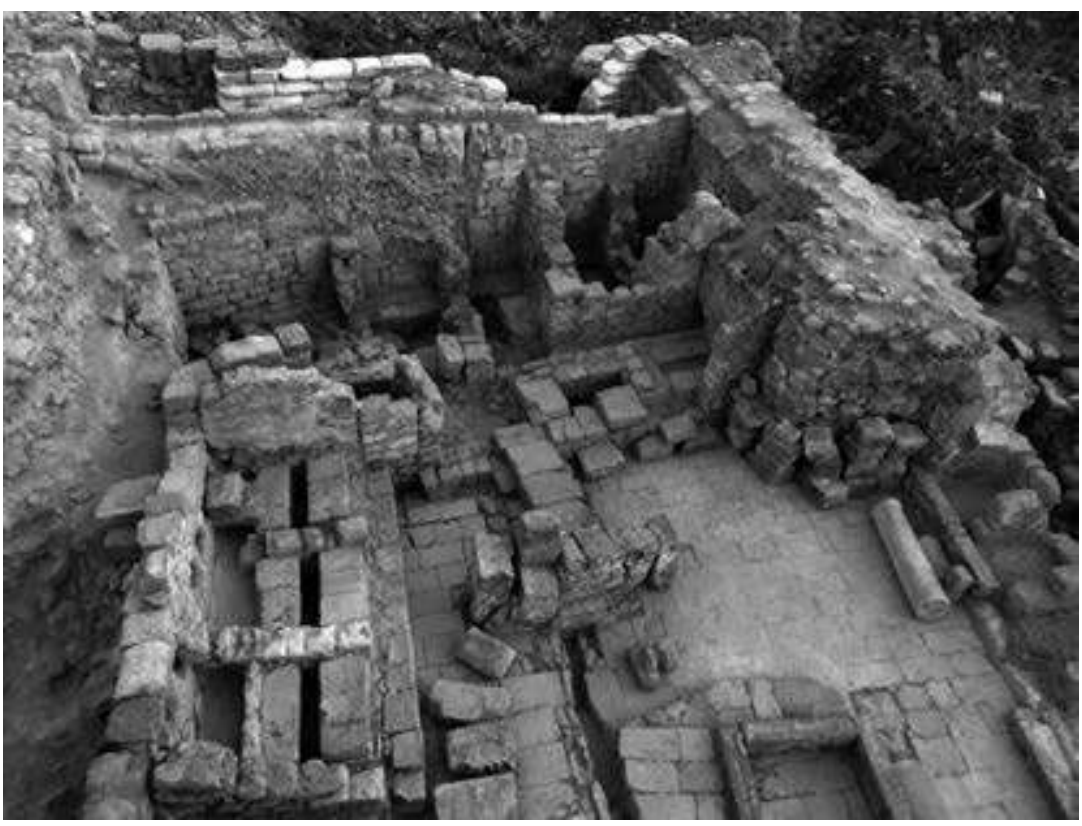

Figure 9. Vue générale de la partie nord, depuis le sud (Cliché Marie-Odile Rousset () Mission archéologique de Tyr) 
Le circuit des adductions et évacuations d'eau est incomplètement conservé, surtout dans l'angle sud-ouest qui a été détruit par la construction de la citerne franque. Cependant, il semble que l'on puisse émettre certaines hypothèses à partir des éléments en place.

Pour la phase I, l'eau qui alimentait les cellules à ablution, du côté est, arrivait du sud, comme l'indique l'enduit conservé à la base du mur, dans l'angle sud-ouest de la cellule T, puis elle circulait de l'une à l'autre. La circulation de l'eau dans la partie nord s'effectuait de l'est vers l'ouest dans le conduit en pierre qui alimentait les petits bassins et qui a sans doute été approvisionné par la canalisation couverte encore observable à l'extérieur du bâtiment, au nord-est. L'évacuation de l'eau grise de la rangée des cellules orientales pouvait s'effectuer dans l'angle nord-est du bâtiment, dans le collecteur des latrines $\mathrm{H}-\mathrm{K}$ et $\mathrm{N}$ qui rejoignait l'égout en activité depuis l'époque romaine ${ }^{15}$.

Un petit réservoir cubique situé dans l'angle sud-est du bâtiment, sans doute destiné à garantir une pression suffisante dans le système hydraulique, pourrait avoir existé dès cette phase, pour alimenter en eau le côté ouest, d'une part, et les compartiments est, d'autre part ( $c f$. infra). Une canalisation est-ouest, prise dans le massif sous l'escalier, au sud, présente deux niveaux successifs d'enduit et pourrait avoir servi à conduire l'eau propre. Le drain ouest, présent dès la première phase de cette partie du bâtiment, aboutissait également au nord-ouest dans l'égout de la pièce $\mathrm{H}$.

\section{Transformations postérieures (phase II)}

Au cours d'une seconde phase, le bâtiment, bien qu'ayant conservé son plan d'origine, a été sensiblement transformé. L'escalier d'accès a été refait, avec une dizaine de marches flanquées de deux murs parallèles (conservés à l'époque des anciennes fouilles : fig. 1 et 2). Une plateforme (1,40 x 0,95 x 0,48 $\mathrm{m}$ de hauteur) a été construite, dans l'angle sud-est de l'édifice, avec deux parements en blocs de grès liés au mortier de chaux. Sur son côté nord, un bassin en marbre, remploi antique, est pris dans la maçonnerie. Le béton de bourrage, de petits galets, est similaire à la couche retrouvée pour l'état final des cellules $\mathrm{Q}$ et $\mathrm{T}$ ( $c f$. infra). Cet ensemble a pu servir à entreposer des vases remplis avec l'eau du bassin.

Six piliers ont été élevés pour soutenir des arcs et voûtes au-dessus des ailes nord et ouest. Ils sont formés de blocs de grande taille $(0,48 \times 0,28 \mathrm{~m})$ posés verticalement, calés par des plus petits et liés à la terre. Deux sont

15. Le collecteur de la rue à colonnade d'axe sud-ouest/nord-est a été étudié lors du sondage 2. Il a été détruit par ce que nous interprétons comme le mur sud (de qibla) de la mosquée (P.-L. GATIER et al., « Mission archéologique... », p. 180-183). Les eaux noires du pavillon d'ablution devaient par conséquent s'évacuer vers la partie nord de ce collecteur ou dans celui de la voie nord-ouest/sud-est. 
construits dans une ancienne cellule du mur est ou contre ce mur. Les autres, espacés en moyenne de $2,75 \mathrm{~m}$, suivent une ligne nord-sud qui correspond à la rupture de niveau du dallage de la pièce A (fig. 9). Ils mesurent environ $0,70 \mathrm{~m} \times 0,48 \mathrm{~m}$. Le premier a été édifié à l'emplacement du retour du mur séparant les pièces I et $\mathrm{N}$. Les deux piliers suivants ont été bâtis sur le dallage, ce qui a entraîné son affaissement à leur base et les a fragilisés. C'est pourquoi, après une première période d'utilisation matérialisée par une couche d'enduit blanc, il a été nécessaire de les pourvoir de contreforts. Ces piliers médians supportaient des arcs dont certains sont encore visibles sur les photographies des fouilles anciennes. L'un d'eux est partiellement conservé dans la maçonnerie nord de la citerne qui a détruit le bâtiment à l'époque franque (fig. 1). Toute la partie ouest, incluant également l'espace de circulation en avant des latrines, a dû alors être couverte par une voûte en berceau portée par trois arcs sur la cour. Une couverture semblable pourrait avoir abrité l'aile nord, soutenue par un seul arc d'une plus grande portée. À l'est, les arcs d'une galerie pourraient également avoir été supportés par les deux colonnes de remploi retrouvées en place lors des premières fouilles, aux angles est du bassin central.

Celui-ci a été construit sur le sol dallé de la cour A (fig. 2 et 3 ; dimensions intérieures : 0,96 x 2,58 x 0,29 m de profondeur) avec des blocs de grès marin. La maçonnerie est protégée par un enduit hydraulique, qui descend jusqu'à la banquette entourant le bassin. Ce dernier est percé de trois conduites aux tuyaux de terre cuite, toutes situées à l'ouest. Dans l'angle sud-ouest, une ouverture carrée de $0,12 \times 0,12 \mathrm{~m}$ creusée dans un bloc de grès, et abritant un tuyau en céramique de $0,06 \mathrm{~m}$ de diamètre, aboutit dans la partie haute du muret et assurait l'alimentation en eau. Une autre se trouve entre deux blocs et se dirige vers la canalisation couverte qui jouxte le bassin à l'ouest ; sa fonction reste indéterminée (évacuation d'un trop-plein ?). La dernière, dans l'angle nord-ouest, au ras du sol, d'un diamètre de $0,06 \mathrm{~m}$, évacuait l'eau vers un orifice situé dans l'angle nordouest de la cour, sous le mur nord.

Durant cette phase, une partie des cellules est, au nord du pilier, a été réutilisée, comme en témoignent les couches d'enduit hydraulique sur leurs sols et murets. Ces espaces étaient voûtés et leur hauteur sous clé atteignait au maximum 1,20 m. Celles situées au sud du pilier ont été agrandies. Désormais au nombre de trois $(\mathrm{R}, \mathrm{S}, \mathrm{T})$, elles mesurent 0,90 x 0,80 m et leur sol est beaucoup plus bas que précédemment. Dans la pièce $\mathrm{R}$, la profondeur atteint $0,53 \mathrm{~m}$ par rapport au point le plus haut de la colonne couchée qui lui sert de seuil (cf. supra). Une ouverture de $0,42 \mathrm{x}$ 0,23 m est ménagée à la base de la partie est du muret sud de séparation, pour que l'eau puisse circuler d'un espace à l'autre, en l'occurrence vers ou depuis la pièce $S$; l'évacuation d'un tel bassin, situé beaucoup plus bas que le sol de la cour A, devait se faire directement dans le collecteur antique 
qui passe sous la pièce Q. Ce dispositif a été rapidement abandonné car l'intérieur ne présente qu'une seule couche d'enduit. L'espace a été comblé avec un béton très dur similaire à celui qui recouvre la plateforme au sud-est ( $c f$. supra), le sol a été rehaussé d'environ $0,50 \mathrm{~m}$ et un muret a été construit sur les colonnes, devant les compartiments, en ménageant un passage d'une dizaine de centimètres pour que l'eau circule d'un espace à l'autre. Dans cette nouvelle configuration, l'eau était évacuée au moyen de tuyaux en céramique pris dans le mortier entre les colonnes et ce muret, disposés dans l'axe d'une cellule sur deux. La pente des sols intérieurs conduisait l'eau vers l'évacuation et, de là, sur le sol de la cour jusqu'à l'égout derrière le mur nord.

Du côté nord, la rangée des pièces $\mathrm{H}-\mathrm{K}$ et $\mathrm{N}$ a été condamnée avec le bouchage des portes et le comblement (au moins partiel) de l'intérieur des pièces par un remblai dans lequel les céramiques les plus récentes sont datées de l'époque fatimide. Une nouvelle ligne d'au moins quatre latrines a été construite en avant de cet espace abandonné ${ }^{16}$. Les latrines $\mathrm{U}$ et $\mathrm{L}$ sont soutenues par un mur est-ouest, qui repose sur le dallage central et passe sur la canalisation nord-sud (fig. 10 et 11). On accède à chaque unité $(1 \mathrm{~m} \times 0,80 \mathrm{~m})$ depuis le sud par une porte de $0,50 \mathrm{~m}$ de largeur. Une rigole d'alimentation en eau propre, en « $U$ », longe le mur nord. Sa jonction avec le sol des latrines est marquée par des blocs posés de chant, de $0,10 \mathrm{~m}$ de large formant un bourrelet de $0,9 \mathrm{~m}$ de hauteur. Ce canal est entièrement recouvert d'au moins deux enduits lissés, rose puis blanc. La pièce la plus orientale de la série a été masquée par un massif plus tardif mais sa présence est attestée par cette rigole qui se poursuit au-delà de la pièce $L$ vers l'est. Son passage sous le muret de séparation est formé par une pierre servant de linteau, posée de biais contre le mur du fond. La fente (est-ouest) qui ouvre sur la canalisation d'évacuation des eaux usées mesure $0,80 \mathrm{~m}$ par $0,20 \mathrm{~m}$. Le dégagement n'a pas permis de déterminer le sens de l'écoulement dans la canalisation mais, d'après son orientation, celle-ci pourrait rejoindre le collecteur qui longe le bâtiment à l'est.

La rangée des latrines occidentales a été construite contre le mur ouest, comme l'indique le plaquage des murets contre les couches d'enduits associées à la première phase d'occupation (fig. 8). Les cabinets devaient à l'origine être au moins sept. En effet, la citerne franque masque le plus méridional, celui de la pièce $\mathrm{C}$ a été démonté lors des fouilles anciennes, probablement pour atteindre les niveaux sous-jacents et le plus septentrional a été détruit par l'aménagement tardif de la pièce $\mathrm{G}$. Des ouvertures quadrangulaires $(0,48 \times 0,30 \mathrm{~m}$ et $0,20 \times 0,20 \mathrm{~m})$ sont aménagées dans les murets, contre le mur ouest afin de laisser passer une rigole d'alimentation

16. L'une d'elles, entre $\mathrm{G}$ et $\mathrm{U}$, a été détruite lors des fouilles antérieures, probablement pour observer la jonction des canalisations. 
en eau propre s'écoulant du sud au nord $(0,35 \mathrm{~m}$ de largeur en moyenne $\mathrm{x}$ $0,24 \mathrm{~m}$ de profondeur). L'appareil des murets, qui reposent sur le drain préexistant de la phase I, comporte deux couches d'enduit blanc lissé portant des graffiti incisés. Les maçonneries des latrines B à $\mathrm{F}$ s'appuient sur le premier enduit. Elles sont construites en encorbellement sur la canalisation d'évacuation, aménageant ainsi la plateforme des latrines $(0,70 \mathrm{~m}$ de largeur $\mathrm{x}$ 0,80 $\mathrm{m}$ de longueur) avec une ouverture longitudinale nord-sud, d'un muret à l'autre, de 0,17 cm de largeur en moyenne. Ces constructions sont

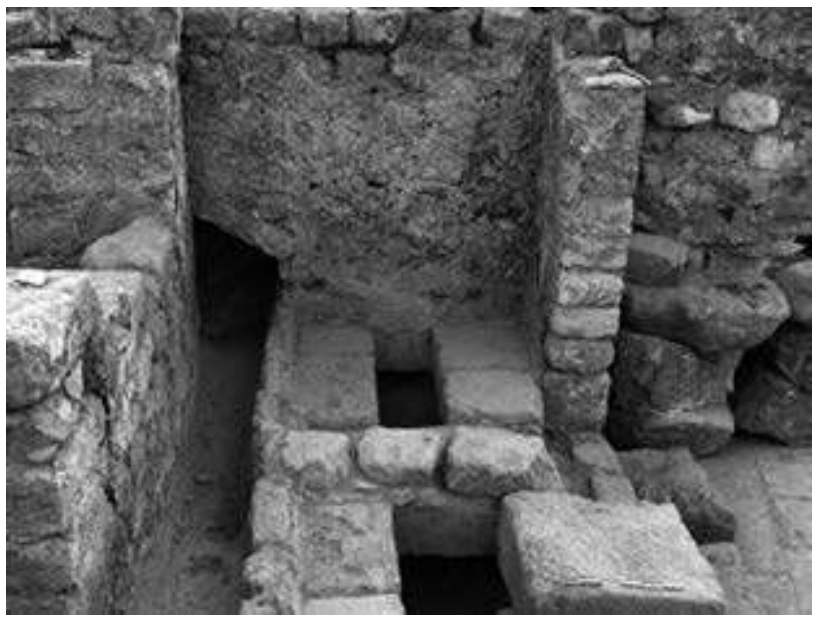

Figure 10. Latrines $U$ et $L$ vues de l'ouest

(Cliché Marie-Odile Rousset $($ C Mission archéologique de Tyr)



Figure 11. Vue verticale des latrines $\mathrm{U}$ et $\mathrm{L}$

(Cliché Marie-Odile Rousset ( C Mission archéologique de Tyr) 
enduites, ainsi que le rebord (0,10 m de largeur x $0,11 \mathrm{~m}$ de hauteur) sur le côté de la rigole. Une banquette a été plaquée contre l'ensemble de ces cabinets, après la transformation de la pièce $\mathrm{G}$ et le bouchage de sa porte. Cette pièce est devenue une citerne surmontant le système d'évacuation des eaux usées.

La hauteur maximale des murets de séparation des latrines n'excédait pas 1,35 $\mathrm{m}$ : l'enduit du parement interne du mur septentrional est continu au-dessus des murets et indique qu'ils ne se poursuivaient pas jusqu'au plafond. À l'image des toilettes publiques actuelles, cela permettait l'aération ainsi qu'un certain contrôle visuel de ces espaces.

Pour la phase II, les pentes des écoulements montrent que l'arrivée de l'eau dans le bâtiment s'effectuait entièrement par le sud, peut-être par une canalisation est-ouest qui passait sur le mur sud. En effet, une trace d'enduit lissé a été observée sur la face supérieure de la partie nord de ce dernier. Il pourrait s'agir là de l'empreinte d'un tuyau, qui aurait conduit l'eau jusqu'au petit réservoir cubique, dans l'angle sud-est du bâtiment. La réserve et le conduit maçonné sont recouverts, à l'intérieur, d'un enduit hydraulique rose à surface grise. De là, l'eau s'écoulait vers la rigole en « $U$ » des latrines ouest, par une canalisation en terre cuite prise dans une maçonnerie constituant, plus loin, l'une des marches de l'escalier. Vers le nord, un canal maçonné entre le mur est et la plateforme jouxtant le réservoir permettait d'alimenter en eau les compartiments orientaux.

À l'autre extrémité du complexe, la pièce $\mathrm{G}$ montre, pour la phase II, trois états de la conduite d'eau propre (fig. 12). Aux deux premiers états des latrines nord $(\mathrm{U}, \mathrm{L})$, elle se présente d'abord sous forme d'une rigole enduite, puis en tuyaux de terre cuite emboîtés qui traversent la pièce en diagonale, pour conduire l'eau pure qui passait dans ou le long du mur ouest vers la partie nord. On retrouve sa trace, vers la porte de l'espace $\mathrm{N}$, sous la rigole postérieure. Maçonné, avec un profil en « $\mathrm{U}$ », le canal d'alimentation en eau propre $(0,24 \mathrm{~m}$ de largeur $\mathrm{x} 0,25 \mathrm{~m}$ de profondeur) du troisième état borde les murs ouest et nord de la pièce $\mathrm{G}$, puis traverse son mur est grâce à un tuyau de terre cuite dont l'embouchure affleurait sur le parement du mur, côté latrines. Ensuite l'eau se déversait de nouveau dans le canal en «U».

Pour la phase II, les évacuations s'effectuaient dans le collecteur antique puis, au nord-est, par une grande canalisation maçonnée rejoignant le grand égout nord-sud jouxtant le bâtiment. Une porte donnait accès à cette canalisation, avec un seuil probablement au-dessus de l'évacuation, bien que celle-ci n'ait pas été atteinte en fouille à cause de l'étroitesse du sondage. L'égout, dans sa partie sud et sur le côté est, est constitué de dalles de grès marin, posées de chant, qui forment un canal de 0,25 à $0,30 \mathrm{~m}$ de large et de $0,35 \mathrm{~m}$ de profondeur, dont le fond est tapissé de pierres. Des traces d'enduit ont été observées à l'état de lambeaux, ainsi que des concrétions liées au passage de l'eau. 
La canalisation construite dans le sol de l'espace central A $(0,20 \mathrm{~m}$ de largeur x 0,34 $\mathrm{m}$ de profondeur) est postérieure à la construction des piliers qu'elle contourne mais antérieure à l'adjonction de leurs contreforts. Elle est délimitée à l'ouest par des blocs verticaux, disposés au droit du dallage qu'elle a cassé dans cette partie. Côté est, le drain utilise le parement de la banquette du bassin et celui du mur nord-sud de la phase I. Ses parois et son fond sont couverts d'un enduit ; sa couverture est faite de grosses dalles. Il se jetait dans l'égout au nord. Il a dû servir à évacuer l'eau du bassin central, lorsque les sols alentours ont été surélevés et que l'eau ne pouvait plus être drainée directement par le sol de la cour.

Enfin, contre le mur est de l'escalier, vers l'entrée, l'embouchure coudée d'une conduite verticale se compose de deux pierres disposées selon un angle de $45^{\circ}$. Un tuyau vertical se trouvait également contre le pilier médian nord et à l'aplomb du drain central. Il semblerait que ces derniers systèmes étaient destinés à évacuer l'eau de pluie plutôt qu'à récolter l'eau du toit.

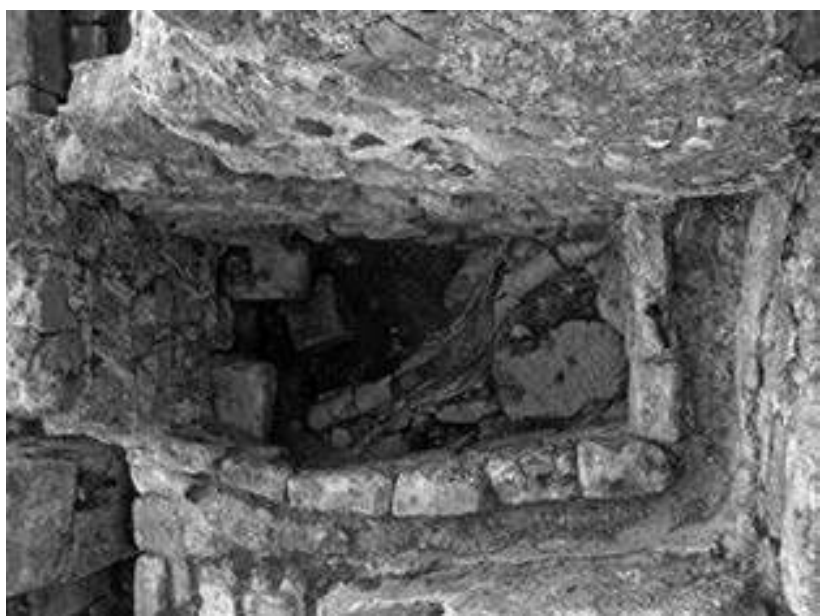

Figure 12. Les canalisations successives dans la pièce $\mathrm{G}$ vues du nord (Cliché Marie-Odile Rousset (C) Mission archéologique de Tyr)

\section{L'exemple de Tyr, un cas particulier?}

Le tour d'horizon des autres lieux d'ablution du Moyen-Orient connus du point de vue de l'architecture ou de l'archéologie est vite accompli. Les installations fouillées (et publiées) sont rares ; on en a expliqué les raisons en introduction. Celles qui offrent une datation, au moins en termes de chronologie relative, le sont encore plus. Néanmoins, 
malgré ces réserves, il est possible d'esquisser et d'expliquer certains des caractères de l'installation de Tyr.

\section{Localisation}

L'élément de comparaison le moins sujet à caution est celui de la localisation des installations sanitaires par rapport aux salles de prière.

À Jérusalem, le $w u d \bar{u}^{\prime}$ était pratiqué dans un espace complètement séparé de la mosquée. Ainsi qu'en témoignent les historiens médiévaux, les inscriptions sur les bâtiments et les édifices eux-mêmes, les installations d'ablution autour et sur le Haram al-Sharīf ont été mises en place ou restaurées par al-Malik al-'Ādil en 1193. Sur le côté ouest de l'esplanade des mosquées, la Bāb al-Mațhara («porte de la purification » ou Bāb al-Siqāya) ouvre sur la Siqāyat al- 'Ādil, où se trouvent encore aujourd'hui des latrines publiques ${ }^{17}$. Accessible également à partir du souk, l'ensemble comprend les lavabos des hommes, au sud, ceux des femmes, au nord, et des bains, à l'ouest. Ces constructions étaient alimentées en eau, entre autres, par une citerne construite à la même époque dans l'esplanade. La seule installation liée aux ablutions sur le Haram al-Sharīf est le Kās, vasque de marbre entourée de sièges, devant la mosquée al-Aqșā. Il y a là, transposés à grande échelle, les éléments que l'on retrouve habituellement dans le complexe religieux, l'ensemble du Haram al-Sharīf correspondant à une mosquée, avec son bassin d'ablution et sa citerne dans la cour et les salles d'eau, toilettes et bains pour les purifications à l'extérieur. Le détail des aménagements d'ablution n'est pas connu car les constructions ont été restaurées à plusieurs reprises ${ }^{18}$.

Dans d'autres cas, la mida'a est comprise dans le même périmètre mais est accessible depuis l'extérieur par un couloir qui l'isole de la cour et de la salle de prière. Il en est ainsi pour les exemples les plus anciens connus archéologiquement pour lesquels les latrines et espaces d'ablution sont situés dans une excroissance de l'angle inférieur gauche du bâtiment, par rapport à la direction de La Mecque ${ }^{19}$. À Ișțabl 'Antar, la petite mosquée de quartier du début de l'époque abbasside est devenue funéraire à l'époque fatimide ; des toilettes ont alors été rajoutées. Pour la grande mosquée de Sīrāf (cinq phases entre la fin du VIII $^{\mathrm{e}}$ et celle du XII ${ }^{\mathrm{e}}$ siècle), la mida'a

17. M. H. Burgoyne, Mamluk Jerusalem : An Architectural Study, Jérusalem, 1987 ; M. HAWARI, Ayyubid Jerusalem (1187-1250) : An Architectural and Archaeological Study, Oxford, 2007, p. 52-56. Le plan des espaces d'ablution antérieurs n'est pas connu : A. KAPLONY, The Haram of Jerusalem 324-1099, Stuttgart, 2002, p. 254-256, p. 400-401.

18. M. HAWARI, Ayyubid Jerusalem..., p. 183.

19. R.-P. Gayraud, X. PeiXOTO, « Isțabl 'Antar (Fostat) 1990. Rapport de fouilles », Annales Islamologiques, 27 (1993), p. 225-232 ; D. WHITEHOUSE, SirafIII.The Congregational Mosque and Other Mosques from the Ninth to the Twelfth Centuries, Londres, 1980. 
s'ouvrait de deux côtés : sur la rue et sur la mosquée. À Sāmarrā, l'un des espaces d'ablution de la Malwiyya se trouvait à proximité immédiate de l'une des portes de la mosquée, dont elle était séparée par un couloir mais à l'intérieur de l'enceinte extérieure du lieu de culte ${ }^{20}$. C'est le cas également des madrasa-s syriennes et des complexes funéraires égyptiens, pour lesquels, lorsqu'elles ont été distinguées, les salles d'ablution sont situées dans un angle de l'édifice.

D'après Doris Behrens-Abouseif, le seul monument mamelouk du Caire qui a conservé une salle d'ablution d'origine est le complexe funéraire du sultan Īnāl (1453-1461) ; toutes les autres ont été refaites à l'époque ottomane ${ }^{21}$. Quelques fouilles archéologiques ont cependant mis au jour des installations plus anciennes, comme à la madrasa de Umm al-Sultan Sha'ban (1368) ${ }^{22}$ et à la madrasa du sultan al-Nāșir Muḥammad (1294-1303 $)^{23}$. Dans ces deux cas, elles ont été installées dans des espaces résiduels du complexe religieux, loin des salles de prière.

Ailleurs, elles jouxtent l'édifice de culte. Ainsi, à Tyr, les installations étudiées se trouvaient à l'extérieur de la cour et des portiques mais à proximité immédiate de l'une des portes d'entrée telles qu'on peut les restituer d'après le plan « classique » de la mosquée fatimide.

Une composante essentielle de la localisation de la mida'a est celle des adductions et évacuations d'eau. Dans certains cas, les textes mentionnent des porteurs d'eau affectés à son fonctionnement, ce qui sousentend qu'il n'y avait pas d'eau courante. Dans d'autres, comme à Tyr, la prise en compte des réseaux antérieurs existant s'est avérée déterminante dans son organisation et sa disposition.

Par sa localisation, la mida'a orientale fait partie intégrante de l'ensemble religieux (sauf dans le cas particulier de Jérusalem). Cette disposition est différente de ce que l'on connaît en al-Andalus et au Maghreb, où les bâtiments d'ablution contemporains (Cordoue : 999, Marrakech : entre

20. M. Al-Samara’I, « Tanqibat Jami` al-Malwiyya $2002 »$, Sumer, 52 (2003-2004), p. 481-492 (p. 484-485).

21. D. Behrens-ABouseIf, Cairo of the Mamluks : A History of the Architecture and Its Culture, Londres/New-York, 2007, p. 267-272.

22. D. ISHAK BAKOUM, «The Madrasa of Umm Al-Sultan Sha'ban before and after Creswell », dans Creswell Photographs Re-Examined, American University in Cairo Press, Le Caire, 2009, p. 99-119 (fig. 4.1).

23. W. MAYer, G. Nogara et P. SPeISER, «Archäologische Untersuchungen und Restaurierungsarbeiten an der Madrasa des Sultan an-Nasir Muhammad in Kairo », Mitteilungen des Deutschen Archäologischen Instituts. Abteilung Kairo, 57 (2001), p. 219238 (fig. 1) ; P. SPEISER, « The Sultan al-Nāṣir Muhammad Madrasah in Cairo : Restoration and Archaeological Investigation », Mamluk Studies Review, 12 (2008), p. 19-221. D'autres espaces d'ablution ont été fouillés dans la madrasa al-Kāmiliyya mais datés de l'époque ottomane : P. SPEISER, « Restaurierungsarbeiten an der Madrasa al-Kāmilīya in Kairo », Mitteilungen des Deutschen Archäologischen Instituts, Abteilung Kairo, 47 (1991), p. 35371. 
1106 et 1120 , Séville : entre 1172 et 1198) sont de véritables monuments, distincts de l'édifice religieux, le plus souvent rejetés de l'autre côté de la rue, en face de l'une des portes ${ }^{24}$. Une autre divergence entre ces deux régions est que la mida'a orientale est parfois semi-enterrée, comme pour Tyr ou pour la mosquée de quartier de Sīrā ${ }^{25}$.

Située au centre de la ville et des quartiers commerçants, l'installation sert aussi de latrines publiques en dehors des heures de prière, dans la mesure où la mosquée reste ouverte en permanence. À Damas ${ }^{26}$ et Alep, des latrines publiques indépendantes des mosquées ont été inventoriées dans les souks. Celles de l'émir Taz à Alep, construites au XII siècle, ont été restaurées en 1357. Elles comportent 23 cellules sur le pourtour d'une grande salle voûtée dont le centre est occupé par un bassin rectangulaire ${ }^{27}$. Cette construction, située sur la voie d'accès à la porte orientale de la mosquée, non loin de celle-ci, pourrait avoir également servi aux ablutions.

\section{Organisation générale de l'édifice}

La répartition générale des différentes composantes des édifices d'ablution semble être la même sur l'ensemble du territoire musulman médiéval : de petits espaces individuels sont répartis sur les côtés d'une cour ou salle centrale, plus grands dans les angles de la pièce. Des drains pour l'évacuation des eaux usées sont aménagés le long des murs extérieurs et rappellent les latrines publiques antiques ${ }^{28}$. Une partie est occupée par les latrines, l'autre par des aménagements pour les ablutions (en général un bassin). L'aspect des lieux varie en fonction de la taille de l'édifice, selon qu'il s'agisse d'une mosquée de quartier (comme à Iștabl 'Antar ou à Sīrāf) ou d'une mosquée congrégationnelle. Dans ce dernier cas, l'architecture

24. A. J. Montejo CóRdOBA, «El pabellón de abluciones oriental de la mezquita aljama de Córdoba correspondiente a la ampliación de Almanzor », Cuadernos de Madinat al-Zahra', 4 (1999), p. 209-231 ; J. Meunié, H. Terrasse, G. Deverdun, Nouvelles recherches archéologiques à Marrakech, Paris, 1957 ; M. VERA REINA, « La mida de la aljama almohade de Sevilla », dans M. VALOR PIECHOTTA éd., El último siglo de la Sevilla islámica (1147-1248), Séville, 1995, p. 161-166.

25. D. WhITEHOUSE, Siraf III..., p. 39-41 : une mosquée de quartier avec un secteur pour les ablutions presque aussi grand que la mosquée elle-même, en contrebas par rapport au sol extérieur, de plan triangulaire.

26. J. SAUVAGET, Les Monuments historiques de Damas, Beyrouth, 1932, n 37, p. 178. Ces latrines sont datées de la première moitié du XIII siècle.

27. J. SAUVAGET, «Inventaire des monuments musulmans de la ville d'Alep », Revue des Études Islamiques, 5 (1931), p. 59-114 (p. 88, ${ }^{\circ} 38$, « Un des seuls exemples anciens de ce genre d'édifices qui se soient conservés en Syrie ») ; J.-C. DAVID et G. DEGEORGE, Alep, Paris, 2002, p. 170.

28. Cf.l'étude de Louise Blanke dans ce même numéro de Médiévales. On retrouve cette disposition en Occident, par exemple à Séville. 
se doit de répondre ponctuellement (par exemple le vendredi) aux besoins d'un grand nombre de personnes en même temps ${ }^{29}$.

\section{Le bassin central}

Un bassin maçonné n'est pas systématiquement présent au centre de l'espace d'ablution. À Sīrāf, c'est une grande citerne rectangulaire qui occupe cette place ${ }^{30}$. Au Caire, il est attesté dès l'époque ayyoubide : celui de la madrasa d'al-Nāṣir Muhammad est de plan carré à l'extérieur, avec des alvéoles semi-circulaires dans les angles qui définissent une cuve octogonale plus profonde ${ }^{31}$. Ce type de bassin est aussi fréquent dans la cour des mosquées et des madrasa-s. Était-il réservé aux ablutions ou pas? Dès lors qu'un système était prévu pour l'évacuation de l'eau qui giclait à l'extérieur, le bassin pouvait être utilisé pour le $w u d \bar{u}^{\prime}$. La fontaine d'eau courante dans le centre de la cour de la mosquée, associée aux « maisons pour se laver » pourvues de latrines à chaque porte d'entrée sont attestées par les textes à La Mecque et à Damas dès l'époque omeyyade ; à Damas, à l'époque de Yāqūt (m. 1225), tous les édifices religieux possédaient un bassin dans la cour ${ }^{32}$. Cependant, son usage pour les ablutions ne se serait développé que tardivement ${ }^{33}$. Quel était le rapport entre le bassin de la mida'a et celui au centre de la cour de l'édifice religieux à l'époque médiévale ? Les deux ont-ils été utilisés simultanément pour les ablutions ? La présence de deux bassins à ablution dans certains édifices se justifiaitelle lorsque des femmes venaient à la mosquée ${ }^{34}$ ? En effet, les sources textuelles montrent qu'elles allaient prier dans les mosquées à l'époque

29. Voir pour l'époque contemporaine A. MOKHTAR, Design Guidelines for Ablution Spaces in Mosques and Islamic Praying Facilities, Sharjah, 2005.

30. D. WhiteHOUSE, Siraf III..., p. 16-19.

31. W. MAYERet al., « Archäologische Untersuchungen... », p. 223-225 ; P. SPEISER, «The Sultan al-Nasir... », p. 207.

32. «Masdjid», EI2 ; dans les madrasa-s ayyoubides et mameloukes de Jérusalem, il n'y a pas de bassin visible dans la cour et aucune mention de latrines ou d'espaces d'ablution sur les plans, ou dans les descriptions. Ceci est dû à la fois à l'état de la recherche et à la conservation des bâtiments, pour la plupart remaniés. M. HAWARI, Ayyubid Jerusalem... ; M. H. BuRGOYNE, Mamluk Jerusalem...

33. Pour L. Hautecendr et G. Wiet, Les Mosquées du Caire, Paris, 1932, p. 270, à partir de l'époque mamelouke bahrite ; pour M. HERZ, «Observations critiques sur les bassins dans les sahns des mosquées », Bulletin de l'Institut Égyptien, 1896 (1897), p. 47-51, à 1'époque ottomane. Dans le cas de la mosquée d'Ibn Țūlūn, bien que l'inscription sur le dôme audessus du bassin de la cour (construit en 1296) fasse allusion à la $4^{\mathrm{e}}$ sourate sur la propreté, M. Herz pense que la profondeur du bassin $(0,85 \mathrm{~m})$ était trop importante et que le jet d'eau au centre prouve le caractère décoratif de l'installation.

34. Lorsqu'elles n'ont pas d'espace dédié, les femmes ont un accès séparé aux espaces d'ablution : A. MOKHTAR, Design Guidelines..., p. 2. 
médiévale même si elles n'y étaient parfois que tolérées ${ }^{35}$. On trouve à Médine un espace spécial pour leurs ablutions dans le portique nord (saqā'if al-nis $\left.\bar{a}^{\prime}\right)$, avec un accès particulier (Bāb al-Nisā') qui aurait été ouvert par le calife 'Umar ${ }^{36}$. Lorsqu'il n'y a pas de salle d'ablution spécifique pour les femmes, celles-ci pourraient-elles avoir utilisé les lieux d'ablutions des hommes ? Ces questions mériteraient une étude spécifique.

Le bassin de Tyr est d'un type différent de ceux évoqués cidessus. Cependant, comme celui d'al-Nāṣir Muhammad, ses côtés sont relativement étroits et pourvus d'un petit rebord. Il n'est donc pas possible de s'y asseoir, ou alors de manière très inconfortable. Une hypothèse serait que son usage pour les ablutions n'était peut-être pas la raison première de son installation. Rectangulaire, peu profond, a-t-il pu servir également pour un usage funéraire, pour les dernières ablutions et la toilette mortuaire ${ }^{37}$ ?

\section{Les latrines}

Les latrines de la phase II de Tyr sont d'un modèle que l'on pourrait qualifier de classique : une plateforme munie d'une fente au-dessus d'un drain. Dans les cas attestés antérieurs à l'époque ottomane, comme à Tyr et dans les deux madrasa-s du Caire, la fente des latrines est parallèle à la porte mais aussi et surtout à l'adduction d'eau qui pouvait facilement être puisée dans la rigole ou le petit bassin ${ }^{38}$. Un tel système présente un autre avantage : lorsqu'aucun système de fermeture n'a été relevé, cela préserve une certaine intimité de l'utilisateur (surtout dans la mesure où ces espaces ne sont pas directement éclairés). À la madrasa du sultan al-Nāṣir Muhammad, des traces de portes en bois ont été relevées ${ }^{39}$. Rien de tel à Tyr, où on peut imaginer éventuellement des rideaux.

Le lavage s'effectuait en puisant l'eau dans la rigole ou le bassin avec la main droite ou un récipient et en utilisant la gauche pour se nettoyer. Cela permettait de ne pas souiller l'eau qui passait ensuite dans le compartiment voisin. Il semblerait que la rigole maçonnée se soit généralisée après le $\mathrm{XII}^{\mathrm{e}}$ siècle car on la retrouve dans les madrasa-s cairotes. Le système du canal qui aboutit dans une petite bassine rectangulaire est attesté pour la

35. « masdjid», EI2.

36. J. SaUVAGET, La Mosquée omeyyade de Médine, Damas, 1947, p. 117.

37. « djanāza », EI2. Cette possibilité est évoquée par D. WHITEHOUSE, «Excavations at Siraf, Third Interim Report », Iran, 8 (1970), p. 16, pour la mosquée funéraire du site G (fin XIII ${ }^{\mathrm{e}}$ ou XIV $\mathrm{X}^{\mathrm{e}}$ siècle).

38. Les latrines de la mosquée al-Nūri de Hama (1163) et du bimaristan Nūr al-Dīn de Damas (1154 et 1242) ont des fentes dans l'axe du cabinet. Considérées comme « monumental evidence of the high standard of sanitation at that period », par Ernst Herzfeld, elles n'étaient sans doute déjà plus d'origine au moment de sa visite : E. HERZFELD, «Damascus : Studies in Architecture II », Ars Islamica, 10 (1943), p. 13-70 (p. 40-47 et fig. 64).

39. W. MAYERet al., «Archäologische Untersuchungen... », fig. 2. 
phase la plus ancienne de Marrakech, contemporaine de la phase finale de l'ensemble d'ablution de Tyr (antérieur à 1124) ${ }^{40}$.

Les latrines de la phase I sont d'un type moins commun : elles comportent une plateforme et un bac à douche disposés côte à côte dans le sens de la longueur. L'association de ces deux éléments existe ailleurs, en contexte résidentiel omeyyade, mais la plateforme occupe le fond de la pièce et le bac est placé en avant ${ }^{41}$. La présence d'un bac à douche associé à la latrine pourrait s'expliquer par la possibilité d'effectuer la purification majeure, le ghusl, au sein de l'espace d'ablution. Ailleurs, la proximité d'un édifice balnéaire, qui peut se justifier pour des raisons évidentes d'adduction et d'évacuation d'eau communes, permet d'associer dans un secteur restreint purification mineure et majeure ${ }^{42}$.

\section{Les espaces d'ablution individuels}

À ma connaissance, il n'existe pas d'autre exemple de rangée de compartiments individuels ouverts sur l'espace central similaires à ceux de l'aile orientale du bâtiment de Tyr. Il s'agit de bacs recouverts d'enduit hydraulique au bord desquels il était possible de s'asseoir pour faire ses ablutions simples. La cuve profonde du premier état de la phase II montre des traces d'usure de sa paroi côté cour qui prouvent qu'il y a eu des frottements répétés de ce côté. Ces bacs n'ont pas pu être utilisés pour le ghusl car ils ne sont pas assez hauts sous la voûte pour qu'une personne puisse s'y tenir debout et n'ont pas de possibilité de fermeture.

À Sīrāf, des rangées de pièces étroites, probablement pour les ablutions, sont mentionnées pour la mosquée congrégationnelle, sans autres précisions ${ }^{43}$.Pour la madrasa d'al-Nāșir Muhammad, les fouilleurs évoquent une « latrine ou douche 7 » pour la phase ancienne (fin XIII ${ }^{\mathrm{e}}$ siècle) ${ }^{44}$.

De nos jours, le $w u d \bar{u}^{\prime}$ ' se pratique de manière collective, dans des espaces ouverts, au bord de bassins, de lavabos ou rigoles bordées de banquettes avec des robinets. Il faut croire qu'il n'en a pas toujours été ainsi et qu'une pratique individuelle a existé à l'époque médiévale, au moins dans un contexte particulier.

40. J. MEUNIÉ et al., Nouvelles recherches..., p. 15.

41. D. GENEQUAND, Les Établissements des élites omeyyades en Palmyrène et au Proche-Orient, Beyrouth, 2012, p. 137, fig. 130.

42. Cf. supra le cas de Jérusalem. À Sīrāf, la mosquée du souk se trouve non loin d'un bain : D. WhiteHOUSE, Siraf III..., p. 32.

43. Ibid., p. 17-19.

44. W. MAYER et al., « Archäologische Untersuchungen... », p. 224. 
On a déjà évoqué l'importance des systèmes hydrauliques préexistants dans la localisation des salles d'ablution ( $c f$. supra). Mais quelle eau était utilisée pour les ablutions ? D'après la tradition, la plus pure est l'eau de pluie, à condition qu'elle soit stockée dans un réservoir suffisamment grand, suivie par l'eau de source, l'eau des puits étant à éviter pour cet usage ${ }^{45}$. À Tyr, les deux premières ont pu être utilisées : plusieurs citernes existent à proximité de la zone d'ablution ainsi que de nombreuses canalisations dont l'étude est en cours ; l'eau de source était encore acheminée à l'époque médiévale par les aqueducs antiques et l'usage de l'eau de mer n'est pas exclu car elle ceignait la ville ${ }^{46}$.

Au Caire, l'alimentation en eau de la salle d'ablution de la madrasa du Sultan al-Nāṣir Muhammad n'aurait pas été continue. Comme à Tyr et à Marrakech, un petit réservoir se trouvait en hauteur dans un angle du bâtiment, sans doute pour maintenir la pression dans les canalisations ${ }^{47}$. Là aussi il y avait un double système de fourniture d'eau : par des canalisations en terre cuite et des rigoles enduites qui couraient le long des murs extérieurs des latrines et par des cruches qui permettaient de puiser dans un petit bassin à côté de l'entrée.

Le recyclage des eaux souillées par les ablutions (eaux grises) qui étaient déversées sur le sol de la cour permettait de la maintenir propre. Leur évacuation par les drains, comme l'eau de pluie provenant des toits à la phase II augmentait le flux qui assurait le nettoyage des latrines installées en fin de circuit et des égouts.

On sait que les questions de détail relatives à la purification ont donné lieu à de nombreuses controverses, notamment entre les différentes écoles coraniques. Est-il possible que les traces matérielles conservées à Tyr soient une traduction architecturale du rite qui y prévalait ? Serait-il possible que les différences entre les établissements d'ablution les mieux connus relevant des rites sunnites et celui de Tyr, d'époque fatimide, donc plus susceptible de relever du rite chiite, s'expliquent ainsi ? L'archéologie pourrait apporter une contribution significative dans la mesure où l'on ne sait encore que peu

45. «wudū" «, EI2.

46. Information orale de Yassir Benhima. Une machine élévatoire aurait alors été nécessaire.

47. W. MAYER et al., «Archäologische Untersuchungen... », p. 228 ; J. MEUNIÉ et al., Nouvelles recherches..., p. 18. 
de choses sur les pratiques et les interprétations médiévales chiites de la loi islamique en matière de rituel de purification ${ }^{48}$.

C'est l'une des nombreuses pistes de recherche ouvertes par cet exemple, qui pose plus de questions qu'il n'en résout. La tentative de synthèse présentée dans cet article montre que plusieurs caractéristiques de l'installation de Tyr sont originales dans le contexte actuel de la recherche sur les salles d'ablution. Cependant, nul ne doute que seules la fouille, l'analyse et la publication de nouveaux exemples pourront étayer ce corpus encore trop faible. De véritables conclusions ne pourront venir que de la confrontation des sources matérielles et textuelles avec une étude anthropologique des pratiques.

\section{Marie-Odile Rousset - CNRS, Archéorient-UMR 5133, MOM}

\section{Latrines et espaces d'ablution dans les mosquées du Proche-Orient médiéval : l'enseignement des fouilles de Tyr}

Pour le croyant, la pratique de la prière est conditionnée par la nécessité de se trouver en état de pureté. Si cette question est souvent évoquée par les sources écrites - notamment les textes juridiques médiévaux -, les sources archéologiques, en revanche, n'ont livré que peu d'informations sur les pratiques liées à la purification rituelle dans le monde islamique. Cet article dresse un état des connaissances sur les espaces d'ablutions et les lieux d'aisance associés aux édifices religieux musulmans en Orient. II présente en détail la salle d'ablution de la mosquée fatimide de Tyr/Sour (Liban), attribuable au $\mathrm{XI}^{\mathrm{e}}$ siècle. Cet essai de synthèse montre une conception ou une mise en œuvre différente des notions d'hygiène et de purification et de leur traduction architecturale, selon les périodes et les régions. Ces différences anthropologiques pourraient-elles être liées aux appartenances dogmatiques?

Ablution - latrines - Liban - mosquée - Sour - Tyr

\section{Latrines and Ablution Places in the Mosques of medieval Middle-East : the Contribution of Tyre Excavations}

For the Believer, the practice of the prayer is conditioned by a personal state of purity. This question is often evoked through the written sources, first of all the medieval legal texts. On the other hand, archaeology delivered only scarce information on the practices linked to the rituals of purification in the Islamic world. This article draws up a summary of our knowledge on the ablution spaces and latrines associated to Muslim religious buildings. It presents in detail the ablution area of the Fatimid mosque of Tyre/Sour (Lebanon), datable to the 11th century. It appeared to be different ways to implement hygiene and purification practices through their architectural design, according to different communities, periods and regions. Could these anthropologic differences be linked to dogmatic/religious rules?

Ablution - Latrines - Lebanon - Mosque - Sour - Tyre

48. R. Gauvain, «A Consideration of Three Recent Approaches to Sunni Purity Law », Islamic Law and Society, 12 (2005), p. 333-393 (p. 393, n. 180 : « As observed, there remains much in particular to add regarding the uses and interpretations of Islamic ritual law among Shi 'i and Sufi scholars »). 
